Journal of Engineering and Applied Sciences 14 (9): 2999-3002, 2019

ISSN: 1816-949X

(C) Medwell Journals, 2019

\title{
Distinct Warp Segmentation of Human Acts
}

\author{
${ }^{1}$ Maram Adil Ali Alaziz and ${ }^{2}$ Suhaam Adnan Abdul Kareem \\ ${ }^{1}$ General Directorate of Education, Ministry of Education, Basrah, Iraq \\ ${ }^{2}$ Department of Graduate Studies, Presidency of University of Baghdad, Baghdad, Iraq
}

\begin{abstract}
The segmentation techniques are built on come together or mathematical index terms which are only interrelated to facts and think about no connection among the info and natural characteristics of human actions. Physical characteristics of human motions are those that can be rapidly professed by humans such as speediness increase of velocity, permanence which are fairly beneficial to distinguish human action division points. The dissection process is efficient in human action and videotape-based information sets. An influence and collection of the methodologies are utilized. To focus on methodologies that intend on labeling of full body actions for instance booting, blowing and gesticulating and we classify them according to method they symbolize the dimensional and chronological configure of actions, how they section actions from an enter pour of image information and find out a vision constant description of activities.
\end{abstract}

Key words: Curvature, dimensionality reduction, human action segmentation, space alignment, information, image

\section{INTRODUCTION}

Movement capture methods have revive be utilized for alphanumeric digitalize archiving of elusive educational properties uniquely habitual tangoes, then the facts gained have been utilized for movement assay of these tangoes. The intention of this investigate is to expand and estimate a way of subdividing the total bulk action facts for the duration of tango into a digit of constituent actions. Division of the body action is a fundamental dispensation assignment for doing many enumerative quantitative and/or qualitative investigates on tango body action. The technique utilizes data in relation to speed of bulk fractions and an alter of a action way and an alter of dimension of the place created by the entire body. The technique planned have been estimated by using tango action information and present-day road Jazz data, then the researches displayed hopeful outcomes. The motion diverse knowledge and action primordial dissection structure for creature action making from action imprisoned data. Advanced dimensional action imprison day are symbolized utilizing a short dimensional illustration by geography conserving system which charts alike action examples to the nearness marks on the short dimensional action diverse. Nonlinear assorted gathering among a short dimensional assorted portrayal and advanced dimensional action information presents a creative replica to produce fresh action string by scheming route on the short dimensional action assorted. The study action primitives by learn short dimensional symbol of corpse bogus through action from action imprisoned data. The features obtained can be owing to unrelated view situation or actions mutual among several deed course. This inspires knowledge with restricted discriminate portions which may help out localise which fractions of the capture on film are noteworthy (Sapienza et al., 2014). Using spatio-chronological composition in the videotape must also develop outcome, now as deformable portion designs hold established well victorious in study credit. Though whereas studiess contain obvious limits which represents we can simply describe a opinion fact for initialisation, three-dimensional place period events are intrinsically vague and luxurious to explain in huge infosets. Hence, it is desired to become accustomed picturesque great designs to exploit infosets without position illustration and to characteristic constant to alters in pretense for instance bag of characteristic and piscator vectors, quite than low point HoG organized. The information of a class-organized dictionary training the reality events of attention. Categorization is then based on renovation where the stamp allocated to every videotape reaches from the best possible thin linear grouping of the educated foundation vectors (action primitives) illustrating the events. A short computational cost Inner-layer model learning the inter-class relationship of the data is added for increasing discriminative power (Castrodad and Sapiro, 2012). In spite of its simplicity and low down computational cost, the technique outperforms before reported results for nearly all standard datasets.

Corresponding Author: Maram Adil Ali Alaziz, General Directorate of Education, Ministry of Education, Basrah, Iraq 
The prophecy of person action is a input release towards clever person machine communication and machine direction-finding (Ferrer and Sanfeliu, 2011). In this chore, we give to a relative revise of numerous forecast purposes that are based on the least curve discrepancy from the present place to all the possible target points, that represents, the points that are pertinent for persons action intentionality. The projected forecaster calculate $\mathrm{s}$, at every break of clock, the track from the current to the target places and creates a forecast of the person action at every break of clock applying only the standard of lowest curve difference. The approach has been authorized in the Edinburgh information studies Forum Walker file. The mid stage, action in the human motion compound and following better-quality chronological sulcus has been concerned in this connection. Though, prior neuroimaging revises have mostly used short, reduced motives and the job of pertinent kinematic limits for the dispensation of person deed remainder uncertain. To observe on this issue by viewing enlarged-duration usual pose of an actor occupied in twofold ordinary actions, to 12 competitors in an fMRI revise beneath inactive screening circumstances. It signify that the kinematic possessions of persons actions are repeatedly supervised throughout daily action as a march to formative events and intention (McAleer et al., 2014). An Adaptable Feature Selection (AFS) process that summaries the features of the inquiry by a linear regression model and various characteristic subsets may be chosen alike to the possessions of the particular inquiry. With a Graded Relevance Feedback (GRF) set of rules, we sophisticated the characteristic separation that augments the recovery presentation alike to the graded relevance of the response examples (Tang and Leung, 2012). By means of a philosophy that describes the reasonable significance among action courses in stipulations of graded relevance, the execution of the AFS-GRF set of rules is calculated and uncovered to outdo further group particular characteristic assortment and action regeneration approaches. The human action appreciation from videotape has been an imperative zone of investigate in PC image. Its appliances bring in observation systems, person PC communications and a variety of genuine planet appliances where one of the artist is a person. Action there in video is changed into a lot of frames and analyzed good. It has been exposed in Fig. 1. A number of re-evaluation chores have been ended by numerous investigators in the circumstance of person deed appreciation. Though, it is establish that there is a hole in text when it approaches to methods of STIP-supported finder for person deed appreciation (Dawn and Shaikh, 2016). A inclusive assess on STIP-based methods for human action recognition. STIP-based detectors are robust in find outing curiosity

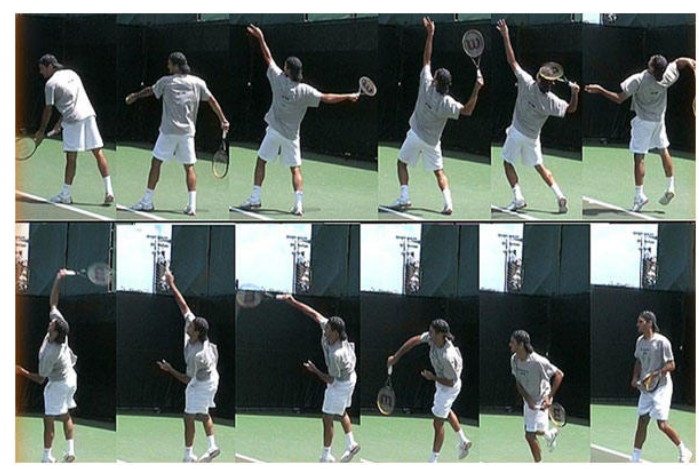

Fig. 1: Video frames of human action

marks from videocassette in spatio-chronological field. This document too condenses linked people textfile helpful for correlating actions of several techniques.

\section{MATERIALS AND METHODS}

Warp space alignment: Picture warping is an exchange which charts all point in single figure flat surface to locations in a subsequent flat surface. It comes up in various picture assay troubles, to remove visual deformations launched by a camcorder or a specific screening viewpoint, to record a picture with a chart or shape or to line up twice or further images. The selection of warp is a concession among a flat warp and one which achieves a fine match. Smoothness can be achieved by assuming a parametric form for the warp or by limiting it applying degree of difference mathematical statement. Matching may be precise by points to bring into arrangement by restricted calculates of link among pictures or by the accident of rims. Parametric and non-parametric methods to warping and corresponding decisive factor are reviewed.

Object segmentation in tagged videos via detection: A lot of observed designs have been projected and accomplish imposing functionings owing to the quick improve of well-explained coaching pictures and device studying concepts. In spite of this, videotape-based SOS it is often not easy to openly teach a administered design while generally motion pictures are feebly explained by labels. A innovative method that accept a dissection by finding structure. The objective detection and segment application are initially generated using the designs coached on motionless pictures which supply of use reminders to generally identify the semantic matter (Kim et al., 2015). Rooted in these suggestions, we suggest a resourceful system to initialize object stalks by answering a combine task trouble. 


\section{RESULTS AND DISCUSSION}

Morphological approach of human detection in video: The configuration may strongly and in reality section semantic things in marked motion pictures, yet, as the picture-supported thing finders offer incorrect suggestions. On several people yardsticks, the future tactic gets considerable developments above the state of the skills. To find out the presence of human in a video view. It obtains usage in determining digit of persons which is found to be a helpful metric in understanding the desired competitors and their communication with the environment.

The foreground is distinguished using Gaussian mix model and is processed to eliminate unnecessary noise by applying apt morphological operations forming a dual image. The foremost blob region is identified using linked component labeling method and averaging methods are employed among clean foreground mask and dual image. In conclusion, edge detection is functional to every processed frame and edge factors in the segmented blob shows the presence of the human in the scene. The qualitative outcomes of the proposed system display improved detection accuracy avoiding missed and bogus detections.

Non linear dimensionality reduction and classification: The dictionary studying process, the neighborhood mass of some potential landmarks on a non-linear various is constrained to keep up the natural local geometric characteristic of the datasets. Meanwhile, to develop the dictionary's discrimination skill, the method can solve the out of sample expansion and large-scale datasets problems effectively. In addition, the analyze results of features, gender and object manner classification display that the researcher's algorithm outperforms a few competing dictionary studying methods.

Temporal human action segmentation: A temporal segmentation and categorization technique that explanation for changeover prototypes among actions of attention. We use this process to mechanically find out most important person deed actions as of motion pictures. A discriminative classifier, (e.g., support vector machine) is helped to realize person deed proceedings and a capable vibrant training system is helped to mutually find out the beginning and conclusion chronological sections of accepted person deeds. The basis dissimilarity from before job is that to start the designing of twice types of occasion changeover message, that is to say happening conversion sections which imprison the happening prototypes among binary successive proceedings of curiosity and happening transition possibilities which design the transition possibilities among the binary proceedings. A categorization method that integrates manifold features, for instance, spectral and spatial message. We apply this method to Manifold Feature Learning via. Rotation (MFL-R) tactic which take on a alternation supported group technique by applying a facts alteration method. Five facts alteration approaches with Principal Component Assay (PCA), Neighborhood Preserving Embedding (NPE), Inear Local Tangent Space Alignment (LLTSA), Linearity Preserving Projection (LPP) and many feature grouping via Multiple Learning and Patch Alignment (MLPA) are used in the MFL-R framework.

Naive Bayes classification: To begin a support for identifying events and things by appraise picture, thing and act supported message from videotape. Hidden Markov designs are mutual with study circumstance to categorize give up proceedings which are collected by a Bayesian classifier to sum up actions. The utilize Bayesian techniques to discernment the group of indefinite objects by estimating noticed events with low down-level, obtained article attributes. Our tactic is apt for pinpointing and categorizing objects under a type of terms together with filled occlusion. To display researches where equally know and before hidden things are accepted applying accomplishment and circumstance a communication. The Naive Bayes classifier, presently practicing a renewal in device knowledge have long been a basis method in message recovery. We look at a few of the differences of Naive Bayes Models helped for manuscript recovery and cataloging, concentrating point on the allocational supposition through about utterance incidence in reports. An approach be composed of designing picture pixels by mechanized mixture of their spectral, textural and extra auxiliary features, division of picture zone employing an repetitive tear and combine system and portraying prospects by decaying them into sample zones and designing the communications among these zones in conditions of their spatial correlations. Naive Bayes classifiers are utilized in the studying of designs for zone division and cataloging.

\section{CONCLUSION}

This manuscript put forward a novel person action division system which explores the person actions from the viewpoint of permanence of the actions. The projected set of rules sections the person action progression build on the quantity of the continuity of the motions and the experiments on different motion sequences have illustrated the strength of the future set of rules. A few of the conversion fasteners among neighboring sections do not bend clearly. The chronological attribute arches created by the set of rules could support the division of 
the action series successfully. It could be observed from the investigational outcome that the planned system will locate out the non-segment positions as the section positions if the stability of the similar activity is in a short grade.

\section{REFERENCES}

Castrodad, A. and G. Sapiro, 2012. Sparse modeling of human actions from motion imagery. Intl. J. Comput. Vision, 100: 1-15.

Dawn, D.D. and S.H. Shaikh, 2016. A comprehensive survey of human action recognition with Spatio-Temporal Interest Point (STIP) detector. Visual Comput., 32: 289-306.

Ferrer, G. and A. Sanfeliu, 2011. Comparative analysis of human motion trajectory prediction using minimum variance curvature. Proceedings of the 6th International Conference on Human-Robot Interaction, March 06-09, 2011, ACM, New York, USA., ISBN:978-1-4503-0561-7, pp: 135-136.
Kim, Y., J. Chen, M.C. Chang, X. Wang and E.M. Provost et al., 2015. Modeling transition patterns between events for temporal human action segmentation and classification. Proceedings of the 2015 11th IEEE International Conference and Workshops on Automatic Face and Gesture Recognition (FG) Vol. 1, May 4-8, 2015, IEEE, Ljubljana, Slovenia, ISBN:978-1-4799-6026-2, pp: $1-8$.

McAleer, P., F.E. Pollick, S.A. Love, F. Crabbe and J.M. Zacks, 2014. The role of kinematics in cortical regions for continuous human motion perception. Cognit. Affective Behav. Neurosci., 14: 307-318.

Sapienza, M., F. Cuzzolin and P.H. Torr, 2014. Learning discriminative space-time action parts from weakly labelled videos. Intl. J. Comput. Vision, 110: $30-47$.

Tang, J.K. and H. Leung, 2012. Retrieval of logically relevant $3 \mathrm{D}$ human motions by adaptive feature selection with graded relevance feedback. Pattern Recognit. Lett., 33: 420-430. 